\title{
OPEN Author Correction: Phylogeny and taxonomic revision of Kernia and Acaulium
}

\section{Lei Su, Hua Zhu, Yongchun Niu, Yaxi Guo, Xiaopeng Du, Jianguo Guo, Ling Zhang \& Chuan Qin}

Correction to: Scientific Reports https://doi.org/10.1038/s41598-020-67347-1, published online 25 June 2020.

This Article contains errors in Table 1.

In the LSU column, the GenBank accession number for Acaulium peruvianum,

“MN991966”

should read:

“MN991967”

In the same column, the GenBank accession number for Kernia hippocrepida was omitted, and should read "MN991966"

(c) The Author(s) 2020 